\title{
Stratifying Monopoles and Rational Maps
}

\author{
Michael Murray
}

Department of Mathematics, I.A.S., The Australian National University, GPO Box 4, Canberra, ACT 2601, Australia.

\begin{abstract}
A stratification of the moduli space of monopoles and of the space of rational maps into a flag variety is presented. It is shown that the map associating a rational map to a monopole preserves these strata. These strata explain some problems in the intepretation of the parameters of the moduli space in terms of superpositions of fundamental monopoles. This interpretation is not valid on the individual strata. The space of fundamental monopoles is described and shown to be the same as the corresponding space of rational maps.
\end{abstract}

\section{Introduction}

Recently Hurtubise (1988) showed that the moduli space of framed $S U(N)$ monopoles, with Higgs field at infinity having distinct eigenvalues, is isomorphic to the space of based rational maps of one dimensional complex projective space, $\mathbb{P}_{1}$, into the space of full flags in $\mathbb{C}^{N}$. This extends the work of Donaldson (1984) who proved the same result for $S U(2)$. These results are particular cases of a general conjecture of Atiyah that the moduli space $\mathscr{M}(X)$ of framed monopoles for a group $K$, with Higgs field at infinity taking its values in an adjoint orbit $X$ of the group on its Lie algebra, is isomorphic to the space $\mathscr{R}(X)$ of based rational maps of $\mathbb{P}_{1}$ into $X$.

The Higgs field at infinity of a monopole defines a class $m$ in $\pi_{2}(X)$ and this labels a decomposition of the moduli space into disconnected pieces $\mathscr{M}(m, X)$. A more precise statement of Atiyah's conjecture is that $\mathscr{M}(m, X)$ is isomorphic to the corresponding connected component $\mathscr{R}(m, X)$ of $\mathscr{R}(X)$. This implies, in particular, that $\mathscr{M}(m, X)$ is connected; a result that has been proven only for $S U(2)$ and $S U(3)$ (Taubes 1985).

It seems likely that the methods of Hurtubise will generalise to $S U(N)$ monopoles with any symmetry breaking at infinity. We examine what this means for the moduli space of monopoles. In particular both $\mathscr{M}(m, X)$ and $\mathscr{R}(m, X)$ have stratifications and we show that these are preserved by the mapping assigning a rational map to a monopole.

In Weinberg 1982 it was suggested that the monopole parameters could be 
explained by regarding them as superpositions of "fundamental monopoles." More recently Bowman (1985) has shown, by counting parameters for the linearisation of Nahm's equations, that this is not always true. The resolution of this apparent contradiction is given by the strata in the moduli space. Bowman's calculations give the dimensions of the various strata, these cannot be explained in terms of fundamental monopoles. Weinberg's calculations are for the whole moduli space, these can be explained in terms of fundamental monopoles. Some examples of this phenomena are given in Sect. 5.

To summarise the rest of the paper. Section 2 explains the general setting and defines the stratification of the moduli spaces, and in Sect. 3 the rational map associated to a monopole is defined. After the stratification of the space of rational maps is defined it is shown that the map from monopoles to rational maps preserves the strata and that the corresponding strata have the same dimension in each case. Lastly in Sect. 5 the structure of the space of fundamental monopoles is examined and Atiyah's conjecture verified in this (trivial) case.

\section{A Stratification of the Moduli Space of Monopoles}

Consider a monopole $(A, \Phi)$ for the group $S U(N)$. It consists of a 1 -form $A$ on $\mathbb{R}^{3}$ taking values in the Lie algebra $s u(N)$ of $S U(N)$ and a Higgs field $\Phi$ which is a function also taking values in $s u(N)$. These satisfy the Bogomoln'yi equations (Hitchin 1982), but it is enough for our purposes to know that the Higgs field, up to gauge transformations, has a limit at infinity which is a map

$$
\Phi^{\infty}: S^{2} \rightarrow s u(N)
$$

from the two sphere of radial directions emanating from the origin to some adjoint orbit of $S U(N)$. Let the distinct (real) eigenvalues of $-i \Phi^{\infty}$ be

$$
\mu_{1}, \mu_{2}, \ldots, \mu_{q}
$$

with multiplicities

$$
n_{1}, n_{2}, \ldots, n_{q},
$$

so that $\sum n_{i}=N$. In such a case the orbit of $S U(N)$ containing the image of the Higgs field at infinity is

$$
F(n)=\frac{S U(N)}{S\left(U\left(n_{1}\right) \times U\left(n_{2}\right) \times \cdots \times U\left(n_{q}\right)\right)}
$$

which, as a complex manifold, is the space of all flags of subspaces

$$
\{0\}=V_{0} \subset V_{1} \subset V_{2} \subset \cdots V_{q}=\mathbb{C}^{N}
$$

with the dimension of $V_{p} / V_{p-1}=n_{p}$ for all $p=1, \ldots, q$.

The monopole is called framed if the Higgs field at infinity at some base point (say corresponding to the positive $z$ axis) is diagonal with eigenvalues ordered as above. The moduli space of framed monopoles is obtained by quotienting the set of all framed monopoles by the group of gauge transformations which are one at this basepoint at infinity. The moduli space of unframed monopoles is obtained 
by allowing gauge transformations that leave the Higgs field at infinity at this base point unchanged. The moduli space of framed monopoles fibres over the moduli space of unframed monopoles and over an irreducible unframed monopole the fibre is

$$
S\left(U\left(n_{1}\right) \times U\left(n_{2}\right) \times \cdots \times U\left(n_{q}\right)\right) .
$$

The homotopy class or degree of the Higgs field at infinity determines $q-1$ non-negative integers, the magnetic charges $m=\left(m_{1}, \ldots, m_{q-1}\right)$ of the monopole. The eigenspaces of the Higgs field at infinity split the trivial bundle $\mathbb{C}^{N} \times S_{\infty}^{2}$ into a direct sum

$$
W_{1} \oplus \cdots \oplus W_{q},
$$

where $W_{j}$ is the $i \mu_{j}$ eigenspace of $\Phi^{\infty}$ and hence has dimension $n_{j}$. The magnetic charge $m_{j}$ is the chern class of the determinant line bundle of $W_{1} \oplus \cdots \oplus W_{j}$. The connection at infinity defines a holomorphic structure on each of the $W_{j}$ and as, by Grothendieck's theorem, any holomorphic vector bundle on $\mathbb{P}_{1}$ splits into line bundles we have

$$
W_{j} \simeq \mathcal{O}\left(k_{n_{1}+\cdots+n_{j-1}+1}\right) \oplus \mathcal{O}\left(k_{n_{1}+\cdots+n_{j-1}+2}\right) \oplus \cdots \oplus \mathcal{O}\left(k_{n_{1}+\cdots+n_{j}}\right)
$$

for each $j=1, \ldots, q$ and some integers $k_{1}, \ldots, k_{N}$.

The magnetic charges are therefore

$$
\begin{gathered}
m_{1}=k_{1}+k_{2}+\cdots+k_{n_{1}}, \\
m_{2}=k_{1}+k_{2}+\cdots+k_{n_{2}}, \\
\vdots \\
m_{q-1}=k_{1}+k_{2}+\cdots+k_{n_{q-1}},
\end{gathered}
$$

and the sets of integers

$$
\begin{aligned}
K_{1} & =\left\{k_{1}, \ldots, k_{n_{1}}\right\}, \\
K_{2} & =\left\{k_{n_{1}+1}, \ldots, k_{n_{1}+n_{2}}\right\}, \\
& \vdots \\
K_{q} & =\left\{k_{n_{1}+\cdots+n_{q-1}+1}, \ldots, k_{N}\right\}
\end{aligned}
$$

are called the holomorphic charges of the monopole. Notice that because of the degeneracy of the eigenvalues of the Higgs field the ordering inside each of these sets is not well determined. We shall choose to order them so that

$$
\begin{aligned}
k_{1} \leqq k_{2} \leqq & \cdots \leqq k_{n_{1}} \\
k_{n_{1}+1} \leqq & \cdots \leqq k_{n_{1}+n_{2}} \\
& \vdots \\
k_{n_{1}+\cdots+n_{q-1}+1} \leqq & \cdots \leqq k_{N} .
\end{aligned}
$$

We shall denote by $\mathscr{M}(m, F(n))$ the moduli space of framed monopoles whose Higgs field at infinity takes values in $F(n)$ and whose magnetic charges are $m=\left(m_{1}, \ldots, m_{q}\right)$. It is expected that these manifolds are connected. 
In the next section we shall see that the holomorphic charges satisfy

$$
m_{p}+k_{i} \geqq 0
$$

for any $p=0, \ldots, q-1$ and $i=n_{1}+\cdots+n_{p}+1, \ldots, n_{1}+\cdots+n_{p}+n_{p+1}$. We shall always make the convention that $m_{0}=m_{q}=0$. Let $\Pi(m, n)$ be all collections $k=\left(K_{1}, \ldots, K_{n}\right)$ of sets of integers satisfying the conditions (2.8), (2.9) and (2.11).

The stratification of the moduli space of framed monopoles is

$$
\mathscr{M}(m, F(n))=\bigcup_{k \in \Pi(m, n)} \mathscr{M}_{k}(m, F(n)),
$$

where $\mathscr{M}_{k}(m, F(n))$ is the space of monopoles with holomorphic charge given by $k$.

As an example consider "projective monopoles" or elements of $\mathscr{M}\left(m, \mathbb{P}_{N-1}\right)$. In this case the magnetic charge $m$ is a single integer and $q=2$ with $n_{1}=1$ and $n_{2}=N-1$. These set $\Pi(m, n)$ is

$$
\left\{\{m, 0\},\{m-1,1\},\{m-2,2\}, \ldots,\left\{m-\left[\frac{m}{2}\right],\left[\frac{m}{2}\right]\right\}\right\} .
$$

\section{The Rational Map of a Monopole}

Using the twistor methods of Hitchin (1982) (see also Murray 1985) each monopole determines a holomorphic rank $N$ bundle $E$ on $T \mathbb{P}_{1}$ the tangent bundle to one dimensional complex projective space. Corresponding to our choice of the behaviour of the Higgs field at infinity $E$ has two filtrations by holomorphic subbundles

$$
\{0\}=E_{0}^{+} \subset E_{1}^{+} \subset E_{2}^{+} \subset \cdots \subset E_{q-1}^{+} \subset E_{q}^{+}=E
$$

and

$$
\{0\}=E_{0}^{-} \subset E_{1}^{-} \subset E_{2}^{-} \subset \cdots \subset E_{q-1}^{-} \subset E_{q}^{-}=E .
$$

The proof in Hitchin 1982 can be adapted to show that the quotients of these bundles are

$$
E_{i}^{+} / E_{i-1}^{+}=L^{\mu_{i}} \otimes \pi^{-1} W_{i}^{*}
$$

and

$$
E_{q-i+1}^{-} / E_{q-i}^{-}=L^{\mu_{i}} \otimes \pi^{-1} W_{i}
$$

for $i=1, \ldots, q$, where $\pi: T \mathbb{P}_{1} \rightarrow \mathbb{P}_{1}$ is the projection. Here $L$ is the exponential bundle introduced in Hitchin 1982.

If we restrict to the zero section of $T \mathbb{P}_{1}$ then the bundle $L$ is trivial as is the bundle $E$. The conditions (2.11) satisfied by the holomorphic charges can be deduced by taking various exterior powers of these bundles and using the two facts that firstly an exterior power of a trivial bundle is a trivial bundle and secondly a line subbundle of a trivial bundle over $\mathbb{P}_{1}$ must have non-positive chern class.

It is useful to look at this also from the point of view of principal bundles as in Murray 1985. The frame bundle $Q$ of $E$ is an $S L(N, \mathbb{C})$ bundle with reductions 
to parabolic subgroups determined by the flags. Decompose $\mathbb{C}^{N}$ as

$$
\mathbb{C}^{N}=\mathbb{C}^{n_{1}} \oplus \mathbb{C}^{n_{2}} \oplus \cdots \oplus \mathbb{C}^{n_{q}}
$$

Call the "standard flag" the flag

$$
\mathbb{C}^{n_{1}} \subset \mathbb{C}^{n_{1}} \oplus \mathbb{C}^{n_{2}} \subset \cdots \subset \mathbb{C}^{N},
$$

and the "anti-standard flag" the flag

$$
\mathbb{C}^{n_{q}} \subset \mathbb{C}^{n_{q}} \oplus \mathbb{C}^{n_{q-1}} \subset \cdots \subset \mathbb{C}^{N} .
$$

Denote by $P$ the stabiliser of the standard flag, $\bar{P}$ the stabiliser of the anti-standard flag and $L$ their intersection. Let $\bar{P}=L \cdot \bar{V}$ be the Levi decomposition of $\bar{P}$, where $\bar{V}$ is the subgroup of $S L(N, \mathbb{C})$ consisting of all matrices with $n_{i} \times n_{i}$ identity matrices along the diagonal, zeroes above these blocks and arbitrary elements below. The flags (3.1) and (3.2) define reductions $R^{+}$and $R^{-}$of $Q$ to $P$ and $\bar{P}$ respectively.

Because the monopole is framed it follows from Hitchin 1982 that over the point on the two sphere at infinity corresponding to the $z$-axis in $\mathbb{R}^{3}$ there is an identification of fibres of the bundles in (2.7). It follows from (3.4) that over the fibre $F \subset T \mathbb{P}_{1}$ of lines parallel to the $z$-axis there is an identification

$$
E_{q-i+1}^{-} / E_{q-i}^{-}=L^{\mu_{i}} \otimes \pi^{-1}\left\{\mathcal{O}\left(k_{n_{1}+\cdots+n_{i-1}+1}\right) \oplus \mathcal{O}\left(k_{n_{1}}+\cdots+n_{i-1}+2\right) \oplus \cdots \oplus \mathcal{O}\left(k_{n_{1}+\cdots+n_{i}}\right)\right\} .
$$

(i) Defining the Rational Map. The rational map of the monopole is defined as follows (see also Atiyah and Hitchin 1988 and Hurtubise 1985). First assume that over $F \simeq \mathbb{C}$ we have a trivialization of $E$ so that the minus flag is mapped to the anti-standard flag in $\mathcal{O}^{N}$. This can be achieved because we can just trivialize $R^{-}$. Moreover asume that under the identification (3.8) coming from the framing this trivialization maps to a given trivialization. Call such trivializations admissible. These constraints mean that an admissible trivialization is determined up to multiplication by holomorphic maps of $\mathbb{C}$ into $\bar{V}$. With respect to any trivialization the plus flag defines a rational map from $\mathbb{C}$ into $F(n)$ the space of all flags of subspaces

$$
\{0\}=V_{0} \subset V_{1} \subset V_{2} \subset \cdots V_{q}=\mathbb{C}^{N}
$$

with the dimension of $V_{p} / V_{p-1}=n_{p}$ for all $p=1, \ldots, q$. In this context we shall use rational as a synonym for holomorphic. By acting on the standard flag we obtain an identification

$$
F(n)=S L(N, \mathbb{C}) / P .
$$

We want to prove that there is precisely one admissible trivialization such that the map $f: \mathbb{C} \rightarrow F(n)$ induced by the plus flag extends to a rational map of all of $\mathbb{P}_{1}$ into $F(n)$ mapping the point at infinity to the standard flag. This rational map we will call the rational map of the monopole.

First let us prove uniqueness. Indeed if we have another admissible trivialisation with this property then we get a different map $v f$ for some map $v: \mathbb{C} \rightarrow \bar{V}$. The subgroup $\bar{V}$ acts freely on a neighbourhood of the standard flag and sweeps out 
an open dense subset of $F(n)$. The complement of this is the union of $q-1$ subvarieties of codimension 1 . The results of Murray 1985 can be extended to this case to show that where the map intersects these subvarieties is the intersection of the spectral curves of the monopole with $F$. This intersection is contained in a closed ball $K$ containing the origin of $\mathbb{C}$. Outside of $K$ the maps $f$ and $v f$ into $F(n)$ have image in the $\bar{V}$ orbit of the standard flag and can therefore be regarded as maps into $\bar{V}$. It follows that the map $v$ extends to a map of all of $\mathbb{P}_{1}$ into $\bar{V}$ and takes the value 1 at infinity. However $\bar{V}$ is a subvariety of $\operatorname{SL}(N, \mathbb{C})$ which is a subvariety of $\mathbb{C}^{N^{2}}$ so that this map must be constant and therefore identically equal to 1 .

Now we prove existence. Choose any admissible trivialization and let

$$
f: \mathbb{C} \rightarrow F(n)
$$

be the map defined by the plus flag. Then, as above, $f$ defines a map

$$
\tilde{f}: \mathbb{C}-K \rightarrow \bar{V},
$$

and this can be regarded as the transition function for a holomorphic $\bar{V}$ bundle on $\mathbb{P}_{1}$.

Such a bundle is trivial. Indeed because $\bar{V}$ is unipotent this bundle is filtered with quotients which are trivial, say

with

$$
W_{1} \subset W_{2} \subset \cdots \mathcal{O}^{N}
$$

$$
W_{1} \simeq \mathcal{O}^{n_{1}}, W_{2} / W_{1} \simeq \mathcal{O}^{n_{2}} \cdots
$$

It follows that $W_{2}$ is an extension of a trivial bundle by a trivial bundle and hence determined by an element in $H^{1}\left(\mathbb{P}_{1}, \mathcal{O}^{n_{1} n_{2}}\right)=0$ so $W_{2}$ must be the split extension and therefore trivial. We can continue up the filtration in this way and show that $W$ is trivial.

Because $W$ is trivial we can write $\tilde{f}$ as a product

$$
\tilde{f}=\tilde{f}_{0} \tilde{f}_{\infty}
$$

where

$$
\tilde{f}_{0}: \mathbb{P}_{1}-\infty \simeq \mathbb{C} \rightarrow \bar{V}
$$

and

$$
\tilde{f}_{\infty}: \mathbb{P}_{1}-K \rightarrow \bar{V}
$$

This factoring of $\tilde{f}$ is unique if we fix $\tilde{f}_{\infty}(\infty)=1$. By applying $\tilde{f}_{0}$ to the chosen trivialization we change the map $f$ to

$$
\left(\tilde{f}_{0}\right)^{-1} f
$$

and this clearly extends to infinity.

This defines the rational map of the monopole. This map can also be defined as "scattering data" in $\mathbb{R}^{3}$ see Hurtubise 1985 and Atiyah and Hitchin 1988.

The collection of $q-1$ non-negative integers which define the degree of a rational map from $\mathbb{P}_{1}$ into $F(n)$ are determined by counting the number of times 
that the image intersects each of the $q-1$ hypersurfaces in the complement to the open orbit of $\bar{V}$. These intersection points are also the intersection points of the spectral curves with the fibre $F$. As in Hitchin and Murray 1988 it is easy to show that the $p^{\text {th }}$ spectral curve intersects the fibre $F$ in $m_{p}$ points. Therefore the rational map of a monopole with charges $m=\left(m_{1}, \ldots, m_{q}\right)$ has degree $m=\left(m_{1}, \ldots, m_{q}\right)$. If we denote by $\mathscr{R}(m, F(n))$ the space of based rational maps of $\mathbb{P}_{1}$ into $F(n)$ of degree $m$ then we have defined a map

$$
\mathscr{M}(m, F(n)) \rightarrow \mathscr{R}(m, F(n)) .
$$

In the next section we define a stratification of $\mathscr{R}(m, F(n))$ and show that the strata of $\mathscr{M}(m, F(n))$ are mapped to the strata of $\mathscr{R}(m, F(n))$. Notice that the group in (2.5) acts naturally on both sides of (3.19) and commutes with this map.

In the case of our example a rational map of degree $m$ into $\mathbb{P}_{N-1}$ is just a collection of $N$ homogeneous polynomials $f_{1}, \ldots, f_{N}$ of degree $m$ which define the map by

$$
\begin{aligned}
\mathbb{P}_{1} & \rightarrow \mathbb{P}_{N-1}, \\
{\left[z_{1}, z_{2}\right] } & \mapsto\left[f_{1}\left(z_{1}, z_{2}\right), \ldots, f_{N}\left(z_{1}, z_{2}\right)\right],
\end{aligned}
$$

where we use the usual homogeneous co-ordinate notation for points in projective space. These polynomials have to satisfy the constraints that they have no common factor and no common zero. Furthermore to be based maps they have to satisfy, say $f_{1}(1,0)=1$ and $f_{j}(1,0)=0$ for $j=2, \ldots, N$.

\section{The Strata of Rational Maps}

There are $q-1$ natural vector bundles $U_{i}$ defined over the variety $F(n)$ by associating to each flag $\left\{V_{1}, \ldots, V_{q-1}\right\}$ the vector space $V_{i}$. More formally

$$
U_{i} \subset F(n) \times \mathbb{C}^{N}
$$

and

$$
U_{i}=\left\{\left(\left\{V_{1}, \ldots, V_{q-1}\right\}, v\right) \mid v \in V_{i}\right\}
$$

These vector bundles provide a filtration of the trivial bundle

$$
U_{1} \subset U_{2} \subset \cdots \subset U_{q-1} \subset F(n) \times \mathbb{C}^{N} .
$$

If $f: \mathbb{P}_{1} \rightarrow F(n)$ is a rational map then it pulls back each of these bundles to a filtration

$$
f^{-1} U_{1} \subset f^{-1} U_{2} \subset \cdots \subset f^{-1} U_{q-1} \subset \mathbb{P}_{1} \times \mathbb{C}^{N}
$$

of holomorphic bundles on $\mathbb{P}_{1}$. By Grothendieck's theorem a holomorphic bundle over $\mathbb{P}_{1}$ splits as a direct sum of line bundles so we have

$$
f^{-1} U_{i} / f^{-1} U_{i-1} \simeq \mathcal{O}\left(-k_{n_{1}}+\cdots+n_{n_{-1}+1}\right) \oplus \cdots \oplus \mathcal{O}\left(-k_{n_{1}+\cdots+n_{i-1}+n_{i}}\right)
$$

for some collection of integers $k=\left\{k_{1}, \ldots, k_{N}\right\}$. The degree of the map is 
$m=\left(m_{1}, \ldots, m_{q}\right)$ where

$$
\begin{gathered}
m_{1}=k_{1}+k_{2}+\cdots+k_{n_{1}}, \\
m_{2}=k_{1}+k_{2}+\cdots+k_{n_{2}}, \\
\vdots \\
m_{q-1}=k_{1}+k_{2}+\cdots+k_{n_{q-1}}
\end{gathered}
$$

and the same proof used for the monopole bundle in Sect. 3 shows that the collection of integers $k$ satisfies the constraints of (2.11). Hence it is an element of $\Pi(m, n)$. We shall order the $k_{i}$ 's as in (2.10) and call them the holomorphic charges of the map $f$. Define $K_{j}$ as in (2.9). If $k_{i} \in K_{j}$ define the multiplicity $\mu\left(k_{i}\right)$ to be zero if $k_{i}=k_{i-1} \in K_{j}$ and otherwise to be the number of $k_{l} \in K_{j}$ with $k_{l}=k_{i}$.

If we denote by $\mathscr{R}_{k}(m, n)$ the subset of $\mathscr{R}(m, n)$ of maps with holomorphic charges $k$, then we have stratification of the rational maps

$$
\mathscr{R}(m, F(n))=\bigcup_{k \in \Pi(m, n)} \mathscr{R}_{k}(m, F(n))
$$

with the indexing set the same as in the case of monopoles.

Before we show that the map from monopoles to rational maps preserves the strata let us consider another way of understanding the strata. If $W$ is any holomorphic bundle on $\mathbb{P}_{1}$ then it splits as a direct sum

$$
W \simeq \mathcal{O}\left(a_{1}\right) \oplus \cdots \mathcal{O}\left(a_{r}\right)
$$

of line bundles. This splitting is not unique. However if $a_{1}>\cdots>a_{r}$ then the subbundle of chern class $a_{1}$ is uniquely determined. In fact, if there is another subbundle of chern class $a_{1}$ then the inclusion maps composed with the projections on to the various factors of (4.8) defines holomorphic sections of $\mathcal{O}\left(a_{i}-a_{1}\right)$ for $i=1, \ldots, r$. However a line bundle of negative chern class admits only the zero section, so all these maps except the map of the line bundle to $\mathcal{O}\left(a_{1}\right)$ must vanish. If we quotient by this subbundle and repeat the argument then we find that $W$ has a uniquely determined filtration

$$
W_{1} \subset \cdots \subset W
$$

with quotients

$$
W_{1} \simeq \mathcal{O}\left(a_{1}\right), \ldots, W / W_{r-1} \simeq \mathcal{O}\left(a_{r}\right) .
$$

If some of the $a_{i}$ 's are the same there is still a unique filtration but the quotients will no longer be 1 dimensional.

If we apply these ideas to the filtration (4.4) and take into account the ordering (2.11) we see that we can "fill out" this filtration until all the quotients are either one dimensional or direct sums of line bundles of the same chern class. This filtration of the trivial bundle defines a map of $\mathbb{P}_{1}$ into another flag manifold which fibers over $F(n)$ and composition with the projection onto $F(n)$ recovers the original $f$. Moreover all maps into that flag manifold of that particular degree arise in this way because of the uniqueness of the filtrations. If we consider then unbased maps we see that the strata correspond to the maps obtained by composing maps into other flag manifolds with the projections onto $F(n)$. 
For the holomorphic bundle of a monopole the same procedure works. When we fill out the filtrations (3.1) and (3.2) in this way we see that all the holomorphic charges become topological quantities. We can use this larger filtration to construct a rational map as in Sect. 3(i) and the result will be a lift of the rational map we constructed with the original filtration. As the topological charges are preserved by the constructions in 3(i) it follows that the holomorphic charges are preserved also.

This proves that the assignment of a rational map to a monopole (3.19) preserves the strata.

In Bowman 1985 the dimension of various sets of solutions to Nahm's equations (Nahm 1983) are calculated. These correspond to the various monopole strata we have defined. We will calculate the dimension of the strata of rational maps and show that they have the same dimension as the corresponding strata of monopoles. Let us denote $\mathscr{R}_{k}^{f}(m, F(n))$ the strata of free, that is not based, rational maps with $k, m$, and $n$ as above. Let $\hat{k}$ be the sequence of $N-1$ non-negative integers $\hat{k}=\left(k_{1}, k_{1}+k_{2}, \ldots, k_{1}+k_{2}+\cdots+k_{N-1}\right)$ and $F\left(\mathbb{C}^{N}\right)$ denote the variety of full flags in $\mathbb{C}^{N}$. The projection map

$$
\pi: \mathscr{R}^{f}\left(\hat{k}, F\left(\mathbb{C}^{N}\right)\right) \rightarrow \mathscr{R}_{k}^{f}(m, F(n))
$$

is onto and in fact a bijection if the $k_{i}$ are all distinct. If some of the $k_{i}$ in a given set $K_{j}$ are the same, then after filling out the flag as much as possible we have some quotients which are direct sums of line bundles all with this repeated value as the negative of their chern class. A flag in such a quotient is determined by choosing a flag in the fibre at any point and if we make this choice then we can extend to a full flag and hence an element of the fibre of (4.11). So the dimension of the fibre of (4.11) is

$$
\sum_{j} \mu\left(k_{j}\right)\left(\mu\left(k_{j}\right)-1\right)
$$

It is easy to calculate the dimension of the space of all rational maps into the full flags using the Riemann-Roch theorem. If we are looking at maps from $f: \mathbb{P}_{1} \rightarrow G / P$ then an infinitesimal change in $f$ is a vector field along the image or a section of the pullback of the tangent bundle. From the Riemann-Roch theorem we have (for real dimensions)

$\operatorname{dim} H^{0}\left(\mathbb{P}_{1}, f^{-1} T(G / P)\right)=\operatorname{dim} H^{1}\left(\mathbb{P}_{1}, f^{-1} T(G / P)\right)+2 c\left(\operatorname{det} f^{-1} T(G / P)\right)+\operatorname{dim}(G / P)$.

The fact that the group acts on $T(G / P)$ shows that it is ample and therefore $H^{1}\left(\mathbb{P}_{1}, f^{-1} T(G / P)\right)=0$. The chern class of the determinant of the pull-back of the tangent bundle can be calculated by relating it to the determinants of the bundles $U_{i}$ which are known to give rise to the degrees of the map. In the case of the full flags in $\mathbb{C}^{N}$ we have

$$
\operatorname{det}\left(T F\left(\mathbb{C}^{N}\right)=\left(\left(\operatorname{det} U_{1} \otimes \cdots \otimes \operatorname{det} U_{N}\right)^{*}\right)^{\otimes 2} .\right.
$$

Alternatively we can usee the result of Hurtubise (1988) to show that the real dimension is 


$$
\operatorname{dim}\left(\mathscr{R}^{f}\left(\hat{k}, F\left(\mathbb{C}^{N}\right)\right)=4 \sum_{i=1}^{N-1}\left(k_{1}+\cdots+k_{i}\right)+\operatorname{dim} F\left(\mathbb{C}^{N}\right)\right.
$$

So using the fibration (4.11) we have

$$
\begin{aligned}
\operatorname{dim} \mathscr{R}_{k}(m, n) & =\operatorname{dim} \mathscr{R}_{k}^{f}(m, F(n))-\operatorname{dim} F(n) \\
& =\operatorname{dim} \mathscr{R}^{f}\left(\hat{k}, F\left(\mathbb{C}^{N}\right)\right)-\operatorname{dim} F(n)-\sum_{j} \mu\left(k_{j}\right)\left(\mu\left(k_{j}\right)-1\right) \\
& =4 \sum_{i=1}^{N-1}\left(k_{1}+\cdots+k_{i}\right)+\operatorname{dim} F\left(\mathbb{C}^{N}\right)-\operatorname{dim} F(n)-\sum_{j} \mu\left(k_{j}\right)\left(\mu\left(k_{j}\right)-1\right) \\
& =4 \sum_{i=1}^{N-1}\left(k_{1}+\cdots+k_{i}\right)+\sum_{i=1}^{q} n_{i}^{2}-N-\sum_{j} \mu\left(k_{j}\right)\left(\mu\left(k_{j}\right)-1\right) .
\end{aligned}
$$

To connect with formula (3.13) of Bowman 1985 we have to remove the framing which for irreducible monopoles means subtracting $\left(\sum_{i=1}^{q} n_{i}^{2}\right)-1$, the dimension of $S\left(U\left(n_{1}\right) \times \cdots \times U\left(n_{q}\right)\right)$. The dimensions are then the same.

In a similar manner it is possible to calculate that

$$
\operatorname{dim} \mathscr{R}(m, F(n))=2 \sum_{i=1}^{q} m_{i}\left(n_{i}+n_{i+1}\right)
$$

agreeing with the result of Weinberg (1982) for the dimension of $\mathscr{M}(m, F(n))$. This should be the dimension of the open strata, which corresponds to the case where the $k_{i}$ in each $K_{j}$ are as close to being identical as possible. We can check formula (4.16) by naively setting $k_{j}=\left(m_{j}-m_{i-1}\right) / n_{i}$ for all $i=1, \ldots, N-1$, $j=n_{1}+\cdots+n_{i-1}+1, \ldots, n_{1}+\cdots+n_{i}$ and substituting these values into (4.16) to get

$$
\begin{aligned}
& 4 \sum_{i=1}^{N-1}\left(k_{1}+\cdots+k_{i}\right) \\
& \quad=4 \sum_{i=1}^{q} \sum_{j=1}^{n_{i}}\left(k_{1}+\cdots+k_{n_{i-1}}+k_{n_{i-1}+1}+\cdots+k_{n_{q-1}+j}\right) \\
& \quad=4 \sum_{i=1}^{q}\left(n_{i} m_{i-1}+\frac{1}{2} n_{i}\left(n_{i}+1\right)\left(m_{i}-m_{i-1}\right) / n_{i}\right) \\
& \quad=2 \sum_{j=1}^{q} m_{j}\left(n_{j}+n_{j+1}\right) .
\end{aligned}
$$

As Weinberg (1982) has pointed out because the formula in (4.17) is linear in $m$ it is reasonable to interpret an arbitrary monopole as a collection of fundamental monopoles which have only one non-zero charge equal to one. In the last section we investigate these fundamental monopoles and show that their moduli space is the same as the space of corresponding rational maps.

\section{Fundamental Monopoles}

Consider a fundamental monopole, that is one with only one non-vanishing magnetic charge say $m_{p}=1$. Then under the twistor correspondence it is equivalent 
to a bundle $E$ on $T \mathbb{P}_{1}$ with filtrations as in (3.1) and (3.2). Because only the $p^{\text {th }}$ magnetic charge is non-zero we have

$$
E_{i}^{+} \cap E_{q-i}^{-}=0
$$

for all $i \neq p$. By using the filtration of the $W_{j}$ we can fill out the filtrations of (3.1) and (3.2) to obtain

$$
\{0\}=E_{0}^{+} \subset E_{1}^{+} \subset E_{2}^{+} \subset \cdots \subset F_{1}^{+} \subset E_{p}^{+} \subset F_{2}^{+} \subset \cdots E_{q-1}^{+} \subset E_{q}^{+}=E
$$

and

$$
\{0\}=E_{0}^{-} \subset E_{1}^{-} \subset E_{2}^{-} \subset \cdots \subset F_{1}^{-} \subset E_{q-p}^{-} \subset F_{2}^{-} \subset \cdots \subset E_{q-1}^{-} \subset E_{q}^{-}=E .
$$

The quotients of these new bundles are

$$
\begin{aligned}
F_{1}^{+} / E_{p-1}^{+} & \simeq L^{\mu_{p}} \otimes \mathcal{O}^{\oplus n_{p}-1} \\
E_{p}^{+} / F_{1}^{+} & \simeq L^{\mu_{p}}(-1) \\
F_{2}^{+} / E_{p}^{+} & \simeq L^{\mu_{p+1}}(1) \\
E_{p+1}^{+} / F_{2}^{+} & \simeq L^{\mu_{p+1}} \otimes \mathcal{O}^{\oplus n_{p+1}-1},
\end{aligned}
$$

and

$$
\begin{aligned}
F_{1}^{-} / E_{q-p-1}^{-} & \simeq L^{\mu_{p+1}} \otimes \mathcal{O}^{\oplus n_{p+1}-1} \\
E_{q-p}^{-} / F_{1}^{-} & \simeq L^{\mu_{p+1}}(-1) \\
F_{2}^{-} / E_{q-p}^{-} & \simeq L^{\mu_{p}}(1) \\
E_{q-p+1}^{-} / F_{2}^{-} & \simeq L^{\mu_{p}} \otimes \mathcal{O}^{\oplus n_{p}-1} .
\end{aligned}
$$

The subbundles $F_{1}^{+}$and $F_{2}^{-}$are defined by the filtration of $W$ using (3.3) and (3.4) which are, in this case

$$
E_{p}^{+} / E_{p-1}^{+} \simeq L^{\mu_{p}} \otimes(\mathcal{O}(1) \oplus \mathcal{O} \oplus \cdots \oplus \mathcal{O}(-1))
$$

and

$$
E_{q-p+1}^{-} / E_{q-p}^{-} \simeq L^{\mu_{p}} \otimes(\mathcal{O}(1) \oplus \mathcal{O} \oplus \cdots \oplus \mathcal{O}(-1)),
$$

it follows that they are always disjoint so that $E=F_{1}^{+} \oplus F_{2}^{-}$. Similarly we have $F_{2}^{+} \oplus F_{1}^{-}=E$. If we define $\widetilde{E}=F_{2}^{+} \cap F_{2}^{-}$this has constant dimension as the dimension is at least two, but if it was more than two anywhere then $F_{2}^{+} \cap F_{1}^{-}$ would be non-zero. Sö $\widetilde{E}$ is a rank two vector bundle. Moreover

$$
\widetilde{E}_{1}^{+}=E_{p}^{+} \cap \tilde{E} \simeq L^{\mu_{p}}(-1)
$$

and

$$
\tilde{E}_{1}^{-}=E_{q-p}^{-} \cap \tilde{E} \simeq L^{\mu_{p+1}}(1)
$$

are line subbundles of $\tilde{E}$ for similar reasons. Finally because of the splittings in (5.1) we have a decomposition of $E$ as

$$
L^{\mu_{1} \oplus n_{1}} \oplus \cdots \oplus L^{\mu_{p} \oplus n_{p}-1} \oplus \tilde{E} \oplus L^{\mu_{p+1} \oplus n_{p+1}-1} \oplus \cdots \oplus L^{\mu_{q} \oplus n_{q}},
$$

and it is straightforward to check that this is preserved by the real structure. This 
decomposition of the bundle $E$ means that this monopole is determined by a representation $\chi: S U(2) \rightarrow S U(N)$ such that $S U(2)$ is mapped to a $2 \times 2$ block on the diagonal and some suitable $U(1)$ monopoles are added in along the rest of the diagonal. Conversely any such representation determines fundamental monopoles by applying it to a translate of the Prasad-Sommerfield monopole.

Let $X_{p}$ denote the set of all such representations. Then taking account of translations of monopoles we have $\mathscr{M}\left(\delta^{p}, n\right)=\mathbb{R}^{3} \times X_{p}$, where $\left(\delta^{p}\right)=\left(\delta_{1}^{p}, \ldots, \delta_{q}^{p}\right)$ is the vector whose only non-zero component is a 1 in the $p^{\text {th }}$ place. The group

$$
S\left(U\left(n_{1}\right) \times \cdots \times U\left(n_{q}\right)\right)
$$

acts transitively on $X_{p}$, and if we fix an embedding as a base point the isotropy subgroup is

$$
S\left(U\left(n_{1}\right) \times \cdots U\left(n_{p}-1\right) \times U(1) \times U\left(n_{p+1}-1\right) \times \cdots U\left(n_{q}\right)\right) .
$$

It follows that we have

$$
\mathscr{M}\left(\delta^{p}, n\right)=\mathbb{R}^{3} \times \frac{S(U(a) \times U(b))}{S(U(a-1) \times U(1) \times U(b-1))},
$$

where $a=n_{p}$ and $b=n_{p+1}$.

Each such representation defines a map $S^{2}=S U(2) / U(1) \rightarrow F(n)$ and, as we shall see, every based holomorphic line arises as the image of such a map. If we denote by $\mathscr{L}(F(n))$ the based holomorphic lines in $F(n)$ then we have a fibering

$$
\mathscr{M}\left(\delta^{p}, n\right) \rightarrow \mathscr{L}(F(n))
$$

and the fiber is $\mathbb{R}^{3} \times S^{1}$ isomorphic to the moduli space of $S U(2)$ monopoles of charge 1 .

Let us consider holomorphic maps $f$ of degree $\delta^{p}$ into $F(n)$. If we look at the fibering

$$
F(n) \rightarrow F\left(\left(n_{1}, \ldots, n_{p-1}, n_{p}+n_{p+1}, n_{p+2}, \ldots, n_{q}\right)\right)
$$

which just forgets the plane of dimension $n_{1}+\cdots+n_{p}$, then composing such a holomorphic map with this fibering gives a map all of whose degrees are zero. But such a map must be a constant and therefore, because it is based any value of $f$ must agree with the the standard flag except that the plane of dimension $n_{1}+\cdots+n_{p}$ may be different. It suffices then to consider based maps into $G_{a}\left(\mathbb{C}^{a+b}\right)$ of degree 1 where $a$ and $b$ are defined above. The image of such a map is a linear subspace in $G_{a}\left(\mathbb{C}^{a+b}\right)$ and they are all defined as follows. First let $\mathbb{C}^{a}$ be the standard plane in $\mathbb{C}^{a+b}$ which we shall use as a basepoint. Then choose a plane $\pi_{0}$ of dimension $a-1$ contained in $\mathbb{C}^{a}$ and a plane $\pi_{1}$ of dimension $a+1$ containing $\mathbb{C}^{a}$. The set of all $a$ planes containing $\pi_{0}$ and contained in $\pi_{1}$ forms a line in $G_{a}\left(\mathbb{C}^{a+b}\right)$ and all lines arise in this way. The set of all such pairs $\left(\pi_{0}, \pi_{1}\right)$ is a homogeneous space of $S(U(a) \times U(b))$ and so we have a fibering

$$
\mathscr{R}((1),(a, b)) \rightarrow \frac{S(U(a) \times U(b))}{S U(a-1) \times U(1) \times U(1) \times U(b-1)}=\mathscr{L}(F(n)) .
$$

The moduli of monopoles as we have seen also satisfies 


$$
\mathscr{M}(\delta, n) \simeq \mathscr{M}(1,(a, b))
$$

and the map (3.18) assigning to a monopole a rational map defines a commutative diagram

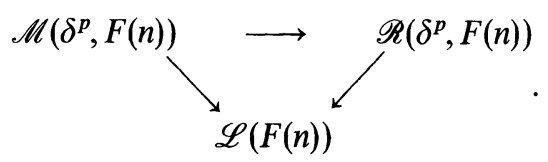

The horizontal map in (5.18) restricted to each fibre is just the map that assigns to each charge $1 S U(2)$ monopole a rational map. Because of the commutativity it follows that this map is a diffeomorphism.

In the example the space of all lines in $\mathbb{P}_{N-1}$ through a fixed point is the projective space $\mathbb{P}_{N-2}$ and the space of rational maps of degree 1 is $\mathbb{R}^{3} \times S^{2 N-3}$. The fibering is

$$
\mathbb{R}^{3} \times S^{2 N-3} \rightarrow \mathbb{P}_{N-2}
$$

with fiber $\mathbb{R}^{3} \times S^{1}$. If we regard $S^{2 N-3}$ as the unit vectors in $\mathbb{C}^{N-1} \simeq \mathbb{R}^{2 n-2}$, then the circle fibre is the set of all unit vectors which lie on the same line in $\mathbb{P}\left(\mathbb{C}^{N-1}\right)=\mathbb{P}_{N-2}$.

If $N=2$ and $m=2$ then there are two strata, the open set labelled by $\{1,1\}$ and of dimension 12 and the strata labelled by $\{2,0\}$. The latter is the set of all rational maps whose image lies in a line in $\mathbb{P}_{2}$. Its dimension is calculated by adding up the 2 real dimensions of the $\mathbb{P}_{1}$ of lines through the base point and the 8 dimensions of the space of all rational maps of $\mathbb{P}_{1}$ to $\mathbb{P}_{1}$ of degree 2 to obtain 10. These parameters can be interpreted as two monopoles in $\mathbb{R}^{3}$ with a vector each on the three sphere. In general this gives $3+3+3+3=12$ parameters, but for the smaller strata the two vectors have to lie in the same $S^{1}$ orbit so there are $3+3$ parameters for the positions, $1+1$ parameters for the points on the orbit and 2 parameters for the choice of orbit giving a total of 10 .

If we consider monopoles of charge 4 then there are three strata, $\{4,0\},\{3,1\}$ and $\{2,2\}$. Using formula (4.16) these have dimensions 18,22 and 24 respectively. The first dimension can be understood as $4 \times 3$ position parameters, 2 for a choice of circle in $S^{3}$ and $4 \times 1$ parameters on that circle giving $12+2+4=18$. The open strata has, of course $4 \times(3+3)=24$ parameters. However there appears to be no simple explanation of the parameters for the middle case, the monopoles would appear to fall into a set of three and one and constraining the three to lie on the one circle produces only 20 parameters.

\section{Conclusion}

It would be interesting to understand the effect of these strata on the dynamics of monopoles (Atiyah and Hitchin 1988). The case of $\mathbb{P}_{2}$ monopoles has been studied by Atiyah and Hitchin (Atiyah private communication). They found that the metric on the moduli space is infinite in directions normal to the strata and therefore monopoles on the strata are trapped.

Further work on these questions is in progress (Hurtubise and Murray 1988). 
Acknowledgement. I would like to thank Howard Garland and Jacques Hurtubise for many useful discussions about monopoles.

\section{References}

Atiyah, M.F., Hitchin, N. J.: The geometry and dynamics of magnetic monopoles. Princeton, New Jersey: Princeton University Press 1988

Bowman, M. C.: Parameter counting for self-dual monopoles. Phys. Rev. D. 32, 6, 1569-1575 (1985)

Donaldson, S. K.: Nahm's equations and the Classification of Monopoles. Commun. Math. Phys. 96, 387-407 (1984)

Hitchin, N. J.: Monopoles and geodesics. Commun. Math. Phys. 83, 579-602 (1982)

Hitchin, N. J., Murray, M. K.: Spectral curves and the ADHM construction. Commun. Math. Phys. 114, 463-474 (1988)

Hurtubise, J. C.: The classification of monopoles for the classical groups. Commun. Math. Phys. 120, 613-641 (1989)

Hurtubise, J. C.: Monopoles and Rational Maps: A note on a theorem of Donaldson. Commun. Math. Phys. 100, 191-196 (1985)

Hurtubise, J. C., Murray, M. K.: Projective monopoles. In preparation

Murray, M. K.: Non-abelian magnetic monopoles. Commun. Math. Phys. 96, 539-565 (1984)

Nahm, W.: Self-dual monopoles and calorons. In: Group theoretical methods in physics. Denado G. et al. (eds). Trieste. Lect. Notes in Phys. vol. 201. Berlin, Heidelberg, New York: Springer 1983

Taubes, C. H.: Min-max Theory for the Yang-Mills-Higgs equations. Commun. Math. Phys. 97, 473-540 (1985)

Weinberg, E. J.: Fundamental monopoles in theories with arbitrary symmetry breaking. Nucl. Phys. B203, 445-471 (1982)

Communicated by A. Jaffe

Received January 26, 1989; in revised form March 20, 1989 